\title{
Proboscis lateralis: A case report of a rare giant craniofacial teratoma in an infant
}

\author{
Sultan Al-Shaqsi ${ }^{1,2}$, Taimoor Al-Bulushi ${ }^{1}$, Qasim Al-Hinai ${ }^{3}$ \\ ${ }^{1}$ Department of Plastic and Reconstructive and Craniofacial Surgery, Khoula Hospital, Muscat, Sultanate of Oman; ${ }^{2}$ Division of Plastic and \\ Reconstructive Surgery, University of Toronto, Toronto, ON, Canada; ${ }^{3}$ Department of Neurosurgery, Khoula Hospital, Muscat, Sultanate of \\ Oman
}

Teratomas can occur in almost any region of the body and are the most common extragonadal germ cell childhood tumors. However, craniofacial teratomas are rare. Craniofacial teratomas can present unique features and cause significant functional and aesthetic concerns. There are complex lesions that can have components intra-cranially and extra-cranially. Therefore, their management requires significant multi-stage multidisciplinary surgical procedures. Herein, we present a case of craniofacial teratoma in a child with the phenotype of proboscis lateralis that highlights some of the pertinent point of the diagnosis and management of congenital neonatal teratomas.

Keywords Teratoma / Skull / Face / Neoplasms
Correspondence: Sultan Al-Shaqsi Division of Plastic and Reconstructive Surgery, University of Toronto, Rotman/Stewart Building, 149 College Street, 5th Floor, Suite 508, Toronto M5T 1P5, Canada

Tel: +1-416-978-8534

Fax: +1-416-978-7316

E-mail:drsultanalshaqsi@gmail.com

The publication of this report was supported by funds from Ibn Sina Pharmacy, Sultanate of Oman.

Received: 1 Dec 2017 - Revised: 26 May 2018 • Accepted: 7 Jun 2018

pISSN: 2234-6163 • elSSN: 2234-6171 • https://doi.org/10.5999/aps.2017.01739 • Arch Plast Surg 2018;45:578-582

\section{INTRODUCTION}

Proboscis lateralis is an abnormal facial growth that can occur in humans [1]. It is a relatively rare congenital facial malformation, in which an anterior "trunk-like" structure projects from the midline face and forehead [2]. Multiple syndromes have been described as associated with this facial deformity, including trisomy 13, holoprosencephaly, cebocephaly, ethmocephaly, and (rarely) cyclopia $[1,3]$.

In the literature, the term "craniofacial teratoma" has also been used to describe the phenotypic features of proboscis lateralis. Furthermore, depending on the exact location, proboscis lateralis can be categorized into extracranial, intracranial, intraorbital, and intraoral subtypes $[1,4]$.

Craniofacial teratomas are rare childhood tumors. It is estimated that such growths account for $2 \%$ of all childhood tu- mors. The incidence of these growths is estimated to be 1:40,000 live births [5]. There is a slight female predilection. Approximately 30 cases of proboscis lateralis have been recorded in the last 150 years [5]. Many such cases have resulted in stillbirth or death shortly after birth from severe disease, which is usually associated with other deformities. The majority of the reported cases have suffered from significant morbidity despite extensive surgical interventions [5]. Craniofacial teratomas can be diagnosed prenatally using ultrasound [6]. Histologically, teratomas are benign lesions, but clinically they can lead to devastating cranial, orbital, and airway deformities. Therefore, the principles of management in such cases include immediate establishment of a secure and patent airway, via tracheostomy if necessary. In addition, early and aggressive surgical resection of the mass is necessary to prevent devastating defects of the field of vision. 
This case report describes a giant craniofacial teratoma in which the facial component of the tumor resembled a typical proboscis lateralis.

\section{CASE}

A 6-month-old baby girl, a product of a monozygous twin pregnancy and vaginal delivery, was referred to the plastic surgery department with a large right-sided craniofacial mass. The mass was noted immediately at birth and had slowly grown. The mass did not cause respiratory obstruction and did not obscure the visual field. Postnatally, the baby was diagnosed with a cardiac ventricular septal defect and a patent ductus arteriosus, for which she underwent an uncomplicated open banding procedure at the age of 2 months. The family reported normal development of the child and no concerns regarding milestone achievements compared to her fully healthy twin sister.

On clinical examination, she had a large right-sided irregular "elephant trunk-like" lesion measuring $10 \times 12 \mathrm{~cm}$ that extended from the right medial canthal area to the right side of her oral commissure. The cephalic component of the mass was soft and compressible, but the caudal segment had a bony block, as shown in Fig. 1. The mass completely obstructed and distorted the right nasal airway. The child was able to open her eyes fully. She did not have any intraoral lesions. Intranasal examination showed a bony bar stemming from the inferior turbinate and obstructing the right nasal airway passage. She had a mild hypertelorism. The rest of the head and neck examination was normal. A detailed pediatric ophthalmological assessment showed normal visual acuity and fields, normal pupillary responses, and normal intraocular findings.
Computed tomography (CT) and magnetic resonance imaging (MRI) showed an isodense mass centered in the floor of the anterior cranial fossa measuring $30 \times 30 \times 26 \mathrm{~mm}$, with herniation of the mass through a right-sided bony defect in the roof of the ethmoidal sinus. The mass then extended to the atretic right-side nasal cavity. There was also a tubular structure in the right atretic nasal airway, with a bony solid block stemming from the right maxillary process into the paracentral proboscis. Right-sided choanal atresia was found, with obliteration of the right-sided nasopharyngeal opening by the bony block. The right-sided maxillary sinus was noted to be hypoplastic. Fig. 2 shows a coronal view of the mass.

The child underwent resection of the lesion, performed by craniofacial surgery and neurosurgery teams. The cranial component of the tumor was approached and exposed through bicoronal bifrontal craniotomy. After opening the dura, the anterior third of the superior sagittal sinus was ligated. This allowed adequate retraction of the anterobasal part of the frontal lobes and exposure of the lesion. The tumor was found to be soft, cheese-like, and vascular.

Thereafter, the extradural part of the residual lesion was pushed through the right ethmoidal bony defect into the right nasal cavity. The bulk of the facial component (proboscis lateralis) was removed via an upper medial canthal incision extending to the lateral nasal and nasolabial skin crease line. This provided adequate exposure for the upper nasal component of the tubular tumor, as shown in Fig. 3. The bony block stemming from the maxilla was trimmed and a right nasal tunnel was created.

Finally, the cranial bones were fixed and bicoronal flaps were closed in layers. Two cranial drains were inserted. The facial incisions were closed after achieving adequate hemostasis. The

\section{Fig. 1. Preoperative views}

(A) Preoperative photo of an infant with proboscis lateralis. (B) Coronal magnetic resonance imaging (MRI) of right-sided proboscis lateralis showing the intracranial component of the tumor (marked T). (C) Sagittal MRI scan of right-sided proboscis lateralis, with the tumor (marked T).
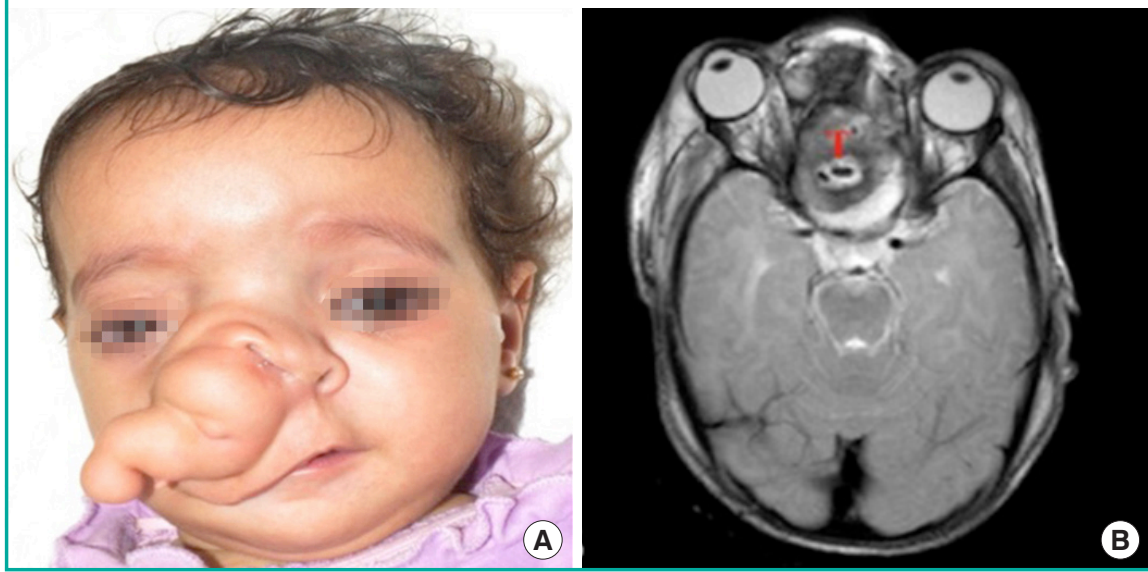

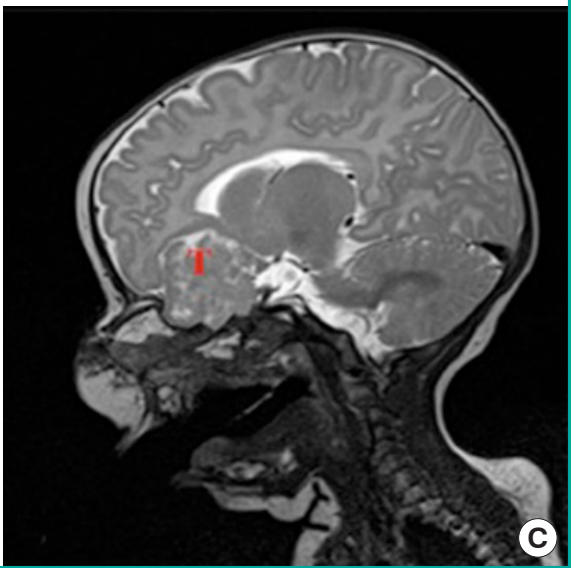




\section{Fig. 2. Operative views}

(A) Cranial skin marking. (B) Bifrontal craniotomy with frontal lobes showing. (C) Markings of the nasal component of the tumor. (D) Resection of the bony block of the nasal component.
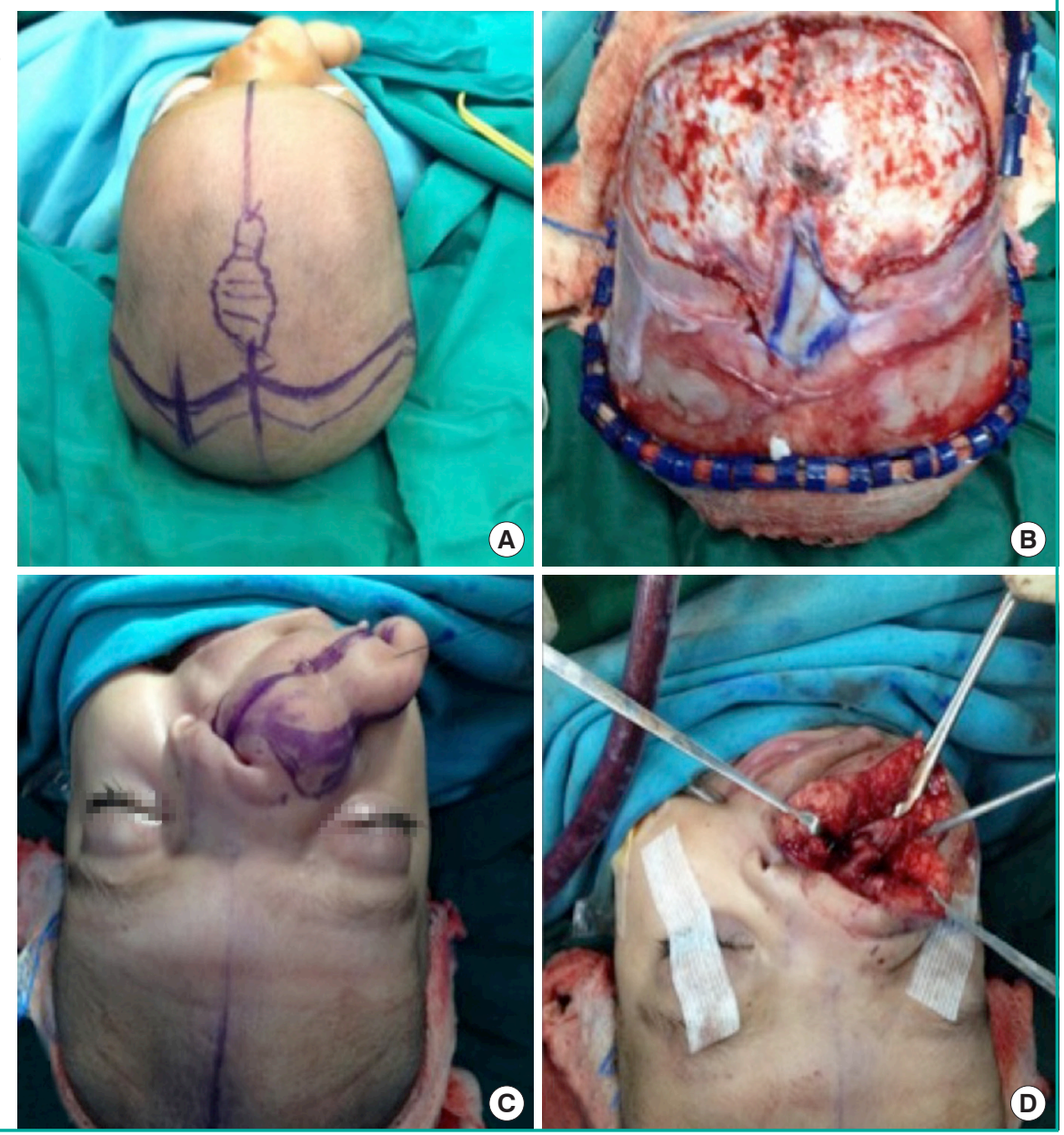

operation lasted 14 hours and the estimated blood loss was $1,000 \mathrm{~mL}$. The child was transfused with eight units of red blood cells, four units of platelets, and four units of fresh frozen plasma. The child tolerated the operation relatively well, with one episode of hemodynamic instability.

Postoperatively, the child was monitored in a pediatric intensive care unit. Her postoperative recovery was complicated by a persistent leak of cerebrospinal fluid (CSF), episodes of respiratory distress, and central diabetes insipidus (CDI). The CSF leak resolved with acetazolamide and conservative management. She was extubated successfully on day 8 postoperatively and discharged home on day 14 . On discharge, she was still suffering from CDI, and was managed by the pediatric endocrinology team.

Histopathological examination of the resected specimens showed fibrofatty tissue with bundles of smooth and skeletal muscle sheets and fibroblastic cells. The overall appearance of the resected tumor was consistent with a cystic craniofacial teratoma.
The child was followed up in the plastic surgery clinic at 6 months postoperatively. Head and facial CT scans showed a small residual intracranial tumor. Clinically, her nasal obstruction had resolved and the right nasal airway remained patent. The child is scheduled for further facial procedures to correct the right-sided nasal deformity at the age of 2 years. Furthermore, she will require a procedure to correct hypertelorism at the age of 8 to 10 years.

\section{DISCUSSION}

The word teratoma is derived from the Greek term teraton, which means "monster." Virchow was the first to use this term in his first publication on tumors in 1863 [7]. There are four basic histological classifications of such tumors in the medical literature. First, desmoids are uncontrolled growths stemming from the epidermal and mesodermal layers of growing tissue [8]. The second type is teratoids, which are poorly differentiated growths that contain ectodermal, mesodermal, and endodermal layers. 
Fig. 3. Postoperative views

(A) Postoperative coronal magnetic resonance imaging (MRI), with the remnant tumor marked T. (B) Postoperative sagittal MRI, with the remnant tumor marked T. (C) Histological slide showing fibrofatty tissue with mature glial cells from a cranial teratoma (hematoxylin and eosin stain, $\times 20)$. (D) Early postoperative photo ( 2 months).
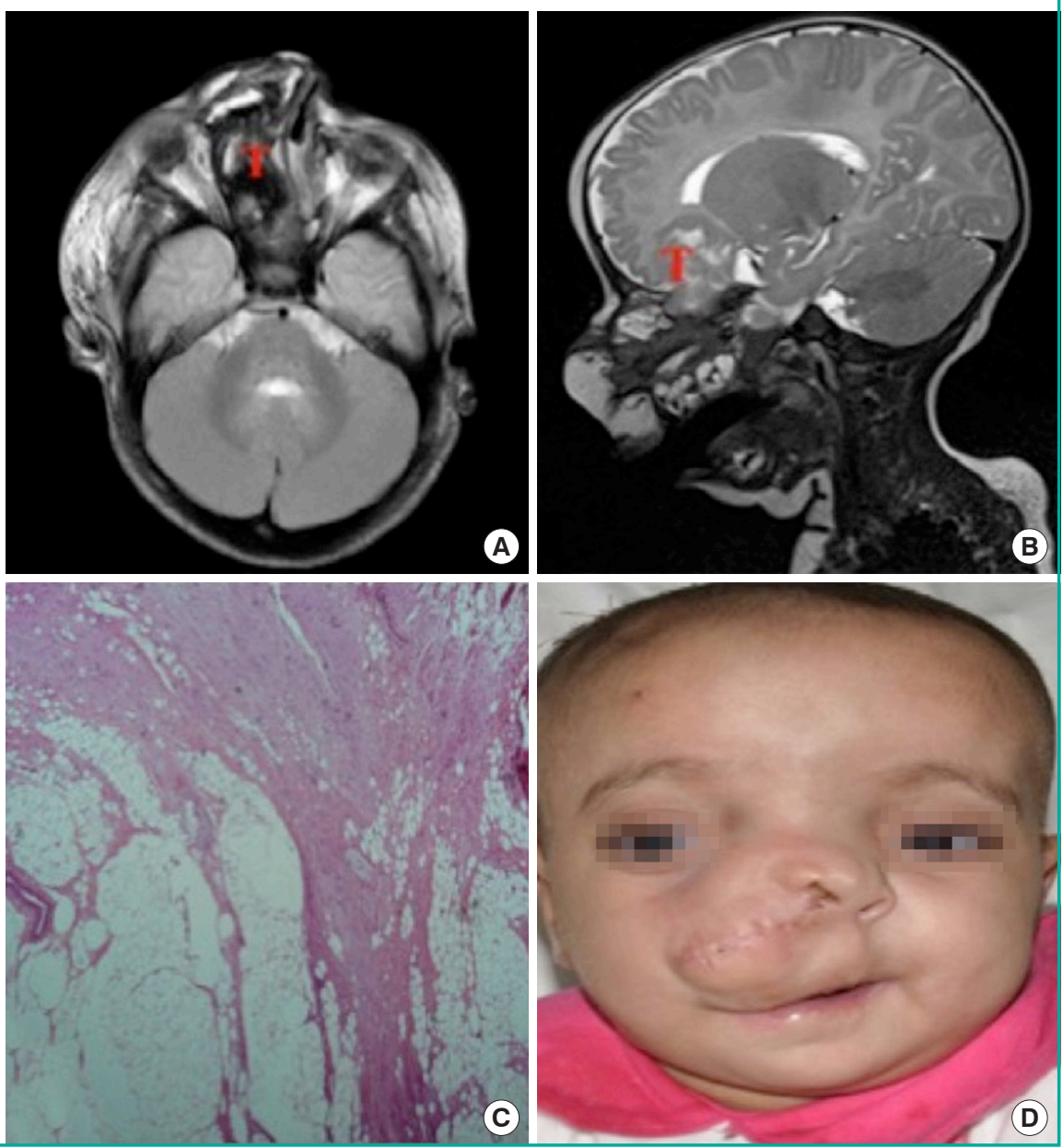

Teratomas are embryologically identical to teratoids. However, teratomas are organized and better-differentiated than teratoids [9]. Finally, epignathi are fully developed organs that are located abnormally [10].

Teratoma is been reported to occur in 1:200,000 live births, of which $2 \%$ are craniofacial teratomas. One phenotype of craniofacial teratoma is proboscis lateralis, which can have both intracranial and extracranial malformations $[11,12]$. Craniofacial teratomas are usually diagnosed prenatally by routine fetal ultrasonography $[2,8]$. Polyhydroamnios is classically a proxy sign that craniofacial malformations are present. The theory is that most craniofacial teratomas lead to obstructions of the oropharyngeal tract; therefore, the fetus is unable to swallow the amniotic fluid and subsequently develops polyhydramnios [4,5]. Furthermore, a few case studies have suggested that calcifications in the head and neck region of newborn babies could indicate the presence of craniofacial teratomas [5]. Moreover, MRI is a useful modality for delineating the extent of such tumors in facial and cranial soft tissues $[13,14]$. Finally, the gold-standard confirmatory test is the histological assessment of intraoperatively resected tissues.
The prognosis of cranial teratomas is difficult to study due to the heterogeneity of these tumors. However, the extent of the intracranial component and its impact on central nervous system development is the major determinant of survival. In other words, teratomas with extensive intracranial components lead to arrest of development of the central nervous system, usually causing intrauterine demise.

There have been several case reports of survival of varying duration in patients with more limited small tumors. One of the earliest reports was published by Whittle, who attempted complete resection of a mature teratoma occupying the entire anterior cranial fossa and left facial region in a 10-week-old child [5]. The child died 6 months later from aggressive recurrence of the tumor, which invaded the brain and led to herniation [5]. Unsurprisingly, the recurrence rate of mature teratomas is lower than that of malignant teratomas due to the maturity of such teratoma lesions $[8,15]$. Nevertheless, the potential for growth of the residual tumor to cause morbidity or death in these cases is still significant.

Advances in microsurgical approaches to the skull base and in- 
fratemporal fossa have expanded the ability of craniofacial surgeons to tackle such difficult and challenging cases, while minimizing morbidity and mortality from these tumors $[3,5,12]$. Despite such advances, no report in the literature has described long-term disease-free survival following radical resection. In addition, perinatal resection has been done successfully. However, those two patients suffered from long-lasting visual and developmental consequences [5].

The case presented in this paper highlights salient features of the surgical management of this rare disease. Of all cases of oropharyngeal teratoma with intracranial extension described in the medical literature, our patient is the first case to be reported from the Middle East. This case demonstrates the complexity and difficulties encountered when embarking on surgical treatment of rare craniofacial conditions.

Craniofacial teratoma is a very rare condition that must be managed by a specialized multidisciplinary team. Combined craniofacial and neurosurgical cooperation during surgical resection is vital to achieve tumor-free survival.

The future plan for this child is to perform serial MRI scans to assess the volume and growth of the remaining teratoma. Furthermore, a second-stage nasal correction is planned at the age of 6 years; this will involve resection of the remaining bony block, rhinoplasty, and strengthening of the alar rims using septal cartilage grafts.

\section{NOTES}

\section{Conflict of interest}

No potential conflict of interest relevant to this article was reported.

\section{Ethical approval}

The study was approved by the Khoula Hospital Ethics Committee (IRB No. KH 302/2016) and performed in accordance with the principles of the Declaration of Helsinki. Written consent was provided by the guardian (father) of the patient.

\section{Patient consent}

The patient provided written informed consent for the publication and the use of her images.

\section{REFERENCES}

1. Martin S, Hogan E, Sorenson EP, et al. Proboscis lateralis. Childs Nerv Syst 2013;29:885-91.

2. Arora G, Arora V, Chawla D. Proboscis lateralis-like appendage: description of a new facial anomaly. Ann Plast Surg 2011;66:357-9.

3. Verma P, Pal M, Goel A, et al. Proboscis lateralis: case report and overview. Indian J Otolaryngol Head Neck Surg 2011; 63(Suppl 1):36-7.

4. Belet N, Belet U, Tekat A, et al. Proboscis lateralis: radiological evaluation. Pediatr Radiol 2002;32:99-101.

5. Aubin A, Pondaven S, Bakhos D, et al. Oropharyngeal teratomas in newborns: management and outcome. Eur Ann Otorhinolaryngol Head Neck Dis 2014;131:271-5.

6. Canan A, Gulsevin T, Nejat A, et al. Neonatal intracranial teratoma. Brain Dev 2000;22:340-2.

7. Jarrahy R, Cha ST, Mathiasen RA, et al. Congenital teratoma of the oropharyngeal cavity with intracranial extension: case report and literature review. J Craniofac Surg 2000;11:10612.

8. Izadi K, Smith M, Askari M, et al. A patient with an epignathus: management of a large oropharyngeal teratoma in a newborn. J Craniofac Surg 2003;14:468-72.

9. Devata S, Chugh R. Desmoid tumors: a comprehensive review of the evolving biology, unpredictable behavior, and myriad of management options. Hematol Oncol Clin North Am 2013;27:989-1005.

10. Berger SL, Kouzarides T, Shiekhattar R, et al. An operational definition of epigenetics. Genes Dev 2009;23:781-3.

11. Sakamoto Y, Miyamoto J, Nakajima H, et al. New classification scheme of proboscis lateralis based on a review of 50 cases. Cleft Palate Craniofac J 2012;49:201-7.

12. Choudhury N, Ghosh T, Mukherjee M, et al. Oropharyngeal true teratoma. Indian J Pediatr 2009;76:747-8.

13. Manchali MM, Sharabu C, Latha M, et al. A rare case of oropharyngeal teratoma diagnosed antenatally with MRI.J Clin Imaging Sci 2014;4:15.

14. Ahmadi MS, Dalband M, Shariatpanahi E. Oral teratoma (epignathus) in a newborn: a case report. J Oral Maxillofac Surg Med Pathol 2012;24:59-62.

15. Kapoor V, Flom L, Fitz CR. Oropharyngeal fetus in fetu. Pediatr Radiol 2004;34:488-91. 